\title{
Healthcare Encounters Data Collection Date Time
}

National Cancer Institute

\section{Source}

National Cancer Institute. Healthcare Encounters Data Collection Date Time. NCI

Thesaurus. Code C123947.

The date and time of healthcare encounters data collection. 